\title{
STATE OF ENERGY ESTIMATION IN ELECTRIC PROPULSION SYSTEMS WITH LITHIUM-SULFUR BATTERIES
}

\author{
Srinivasan Munisamy ${ }^{*}$, Daniel J. Auger ${ }^{1}$, Abbas Fotouhi ${ }^{1}$, Bob Hawkes $^{2}$, and Euthymios \\ Kappos $^{2}$ \\ ${ }^{1}$ School of Aerospace, Transport and Manufacturing, Cranfield University, Cranfield MK43 OAL, UK \\ ${ }^{2}$ OXIS Energy Ltd, Abingdon, Oxford OX14 3DB, UK \\ *srinivasan.munisamy@cranfield.ac.uk; msrinivasan@ieee.org
}

\begin{abstract}
Keywords: LITHIUM-SULFUR BATTERY, NONLINEAR FUNCTION, STATE-OF-ENERGY, STATE-OFCHARGE, NONLINEAR FILTER.
\end{abstract}

\begin{abstract}
Lithium-Sulfur (Li-S) batteries are an emerging and appealing electrical energy storage technology. The literature on the Stateof-charge (SoC) estimation of Li-S is readily available. In real-world, battery operated vehicles and equipment need to monitor the electrical energy. This paper focuses on State-of-Eneergy ( $\mathrm{SoE}$ ) estimation of Li-S battery based electric propulsion system. This paper bridges literature gap of the SoE estimation of Li-S battery. While comparing mathematically, the definition of the SoC and SoE batteries are different. Reviewing the SoC estimation, this paper compares the SoC and SoE estimation for same data set. The challenges in Li-S SoC and SoE estimation include battery modelling and time-varying parameters and nonlinear voltage measurement, which has deeply skewed high-plateau and flatted low-plateau characteristics. Modelling Li-S battery as a Thevenin's equivalent circuit network (ECN), the battery parameters are estimated using Predict Error Minimization (PEM) approach. For estimate SoC and SoE, the extended Kalman filter (EKF) is used. Since the parameters are high sensitive to battery current, the estimators use parameters obtained by polynomial fitting model. A simple switching logic based on SoCmeasurement voltage is used to join the high- and low-plateau. The degree of observability analysis is used to investigate the performance of SoE estimation by the EKF. Using experiment test data, simulation results demonstrate the performance of both SoC and SoE estimators. Results show that the SoE estimation is as close to the SoC estimation.
\end{abstract}

\section{1 Introduction}

Lithium-sulfur (Li-S) battery technology has been active research since last few decade, for instance [1-6, 8]. The principal of Li-S battery chemistry reactions and features studied in [3]. Compared to Lithium-Ion (Li-Ion) and solid state batteries, the Li-S batteries are weightless, high energy storage capacity, more safe, fast charging and less cost. With these note, it may be better choice of energy source to applications where, weight and safety have concern that including electrically propelled aircraft [16] and electric ground vehicles [7]. Battery management system (BMS) are an essential and important component in such applications.

The challenges for battery management system (BMS) community is to develop the state-of charge(SoC), state-of-available power (SoAP) [10], state-ofenergy(SoE)[14], and state-of-health $(\mathrm{SoH})$ algorithms under nonlinear voltage measurement. We need nonlinear estimation algorithms. Literature [1-5] explored on SoC estimation of Li-S battery using Kalman-variant filters [3,4] and adaptive neuro-fuzzy inference system (ANFIS) [5]. In [89, 14] the SoE of Li-Ion explored.

Compared to SoC, which indicates the residual capacity, the SoE provides the residual energy of battery. Power performance of battery due the power fluctuations caused by acceleration, climbing and regenerative braking can be monitored while estimating energy. Compared to lithiumion Li-Ion [7] batteries, the Lithium-Sulfur (Li-S) cells have a distinctive open-circuit voltage (Voc) profile: at high states of charge there is a 'high plateau', starting at around $2.35 \mathrm{~V}$, and at low states of charge there is a flatter 'low plateau' at near constant voltage. This distinctive behaviour results nonlinearity in the system and measurement models of battery.

This paper focus on the SoE estimation of Li-S battery using Extended Kalman Filter (EKF. As literature on Li-S battery's SoE estimation is not available, this work would fill that the gap. This work differs from the literature as follow: In our previous presentation [8], based on Coulomb counting (CC) of SoC formula and the theoretical SoE defined in terms of terminal power $\left(V_{T} I_{l}\right)$, we discussed the how much the SoC of Li-S cells is very close to $\mathrm{SoE}$ of Li-Ion and Li-S. Compared to [8], this paper focuses on EKF based SoE estimation. In addition to compare SoE to the SoC estimation, this work also investigates the impact of $\mathrm{SoE}$ defined as function of remaining power $\left(V_{o c}(S o E) I_{l}\right)$ of Li-S. The EKF based SoC of Li-S [3] developed with a blend function in order to smooth linking of polynomial curve fitting of low and high-plateau. In contrast to [3], this work investigates the EKF based SoE and compared that to SoC estimation.

In this work the SoE estimation of Li-S battery problems are formulated, and designed the EKF for that. The SoE estimation problem formulation is very similar to SoC 
estimation problem except mathematic model as outlined earlier. Modelling Li-S battery as Equivalent Circuit Network $(\mathrm{ECN})$, nonlinear Prediction error minimization (PEM)[3, 12, 17] is used for parameter estimation. The PEM uses a nonlinear grey model of ECN as [3]. While designing EKF for SoE and SoC problems, a simple switching logic based on the empirical SoC over Voc curve is used for high- and lowplateau the priori state and measurement estimation, and Jacobian. Using test result of discharge profile of a sample Li$\mathrm{S}$ cell designed for aerospace propulsion, the EKF based SoE estimation and its comparison to $\mathrm{SoC}$ is demonstrated with simulation. The accuracy of estimation is investigated by analysing degree of observability.

The rest of paper is organised as follow. Section 2 discusses the modelling of battery system and sensor, and estimation problem formulation. Battery parameter estimation and their curve fitting models are presented in section 3. Section 4 presents the EKF based SoC and SoE estimation. The simulation performance of estimators is described in section 5. Finally the conclusions and future research are given in section 6 .

\section{Battery Modelling and Problem Formulation}

Experiment, which is prototype to real-world battery in use of electric vehicles, provides battery current and terminal voltage. Test result of the sample Li-S cell, the current and voltage with time stamp, obtained by laboratory experiment are shown in Fig.1. This data set has the mixed pulses current as Fig.1a, and mixed pulse voltage as Fig 1b. Mixed pulses represent the real-world load variation in electric propelled aircraft.
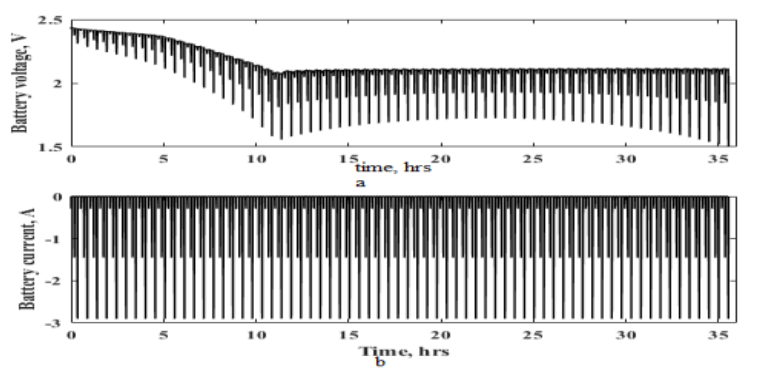

Fig. 1 The experimental test results of Li-S battery: a) Battery terminal voltage, and b) battery current.

The voltage characteristic of battery is highly nonlinear that forces the problem as nonlinear. Having current as input and voltage as output, the battery might be a black box control system as shown in Fig. 2.

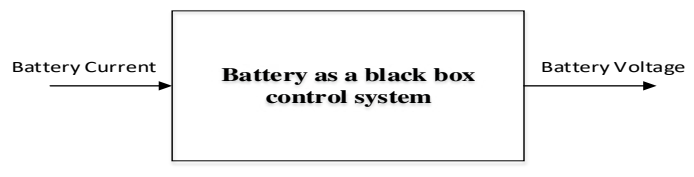

Fig.2 Battery as a black box control system
The battery can be modelled as equivalent circuit network (ECN) model, and then it can be used to estimate the parameters and states of battery.

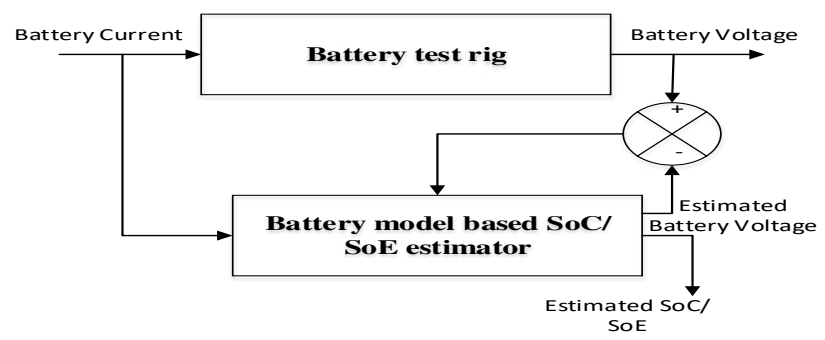

Fig.3 SoC and SoE estimation problem formulation from experiment result.

In this work, the EKF based the SoC and SoE estimation are presented. The EKF for SoC and SoE have designed separately as they are mathematically different. However, the problem formulation for both is very similar. For both estimation problem, the state-space model is formulated as the standard nonlinear functions as follows:

The state equation is

$\dot{x}(t)=f(x(t), u(t), t)+,w(t)$;

with initial condition $x(0)=x\left(t_{0}\right)$. The measurement equation is

$y(t)=h(x(t), u(t), t)+,v(t)$

In above equations, $x(t)$ denotes the state vector, $\dot{x}(t)$ is first derivative of state vector, $f($.$) and h($.$) is state transition and$ measurement function, respectively, of $x(t)$, input $u(t)$ and time $t$, and $w(t) \sim N(0, Q)$ and $v(t) \sim N(0, R)$ is process and measurement noise, respectively.

Both SoC and SoE problem, the input $u(t)$ is battery current $I_{L}$, and the measurement $y(t)$ is battery terminal voltage $V_{T}(t)$ with additive noise $v(t)$. This terminal voltage of battery cell depends upon the current density and can be modelled as a function of polarization voltage. Therefore, the polarization voltage of cell $V_{F}(t)$ is one of the state variable in both SoC and SoE estimation problem. The following subsection discuss the state vector for SoC and SoE estimation.

\subsection{SoC estimation problem.}

The SoC estimation problem is formulated with a state vector $x(t)=\left(\begin{array}{c}x_{\text {soc }}(t) \\ V_{F, \text { soc }}(t)\end{array}\right)$

Where $x_{\text {soc }}(t)$ denotes the SoC, and $V_{F}(t)$ denotes the polarization voltage.

The SoC is defined as [3]

$x_{\text {soc }}(t)=x_{\text {soc }}\left(t_{0}\right)-\int_{0}^{\tau} \frac{I_{L}}{C_{B}} d \tau$

where $x_{\text {soc }}\left(t_{0}\right)$ is the SoC at the time $t_{0}, I_{L}$ is the battery current and $C_{B}$ is the capacity of battery. This empirical evaluation of SoC is referred as Coulomb counting approach. Since this paper uses discrete EKF, the discrete time, the equation (4) can be expressed in discrete-time as

$x_{\text {soc }}(k)=x_{\text {soc }}(k-1)-\frac{I_{L}(k) \Delta t}{C_{B}}$

The $\Delta t$ is the sampling time, and $k$ denotes time index.

The dynamic of polarisation voltage $V_{F}(t)$ can be written as 
The voltage sensor of battery can be modelled as

$y_{\text {soc }}(k)=h\left(x_{\text {soc }}(k), V_{F, s o c}(k), I_{L}(k)\right)+v(k)$

Where

$h()=.V_{T, s o c}=V_{o c v}(s o c)-V_{F}(s o c)-V_{i}(s o c)$

is measured terminal voltage that derived mathematically derived from ECN model given as Fig.4. for simplicity, $I_{L}$ and $T$ are droped in the output voltage.

The first-order differential of $V_{F}(s o c)$ and its discrete version we have used same as [1-3]. The differential of polarization voltage $V_{F}(t)$ is expressed as

$\frac{d V_{F}(t)}{d t}=-\Omega(.) V_{F}(t)+\rho(.) R_{\text {int }}(.) \Omega(.) I_{L}$,

and the measurement model is

$y_{c}(t)=V_{o c v}()-.V_{F}(t)-(1-\Omega().) R_{\text {int }}(.) \Omega(.) I_{L}$

where

$\Omega()=.\frac{1}{R_{F}(.) C_{F}(.)}$

$R_{\text {int }}()=.R_{i}()+.R_{F}($.

$\rho()=.\frac{R_{F}(.)}{R_{\text {int }}(.)}$

Thus the new parameters to the actual ECN parameters are related as [3].

Using measured voltage, the EKF estimates the SoC and polarisation voltage.

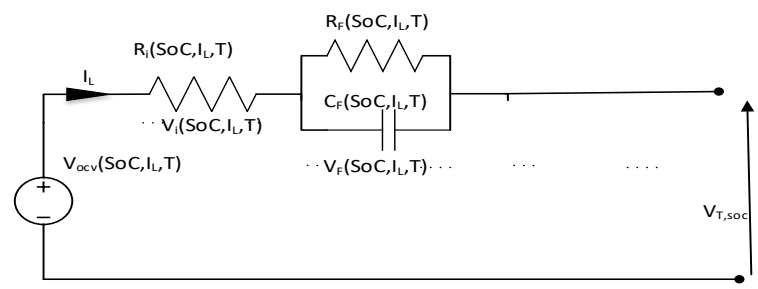

Fig.4 Battery model for SoC estimation

\subsection{SoE estimation problem}

The SoE estimation problem assumes the state-of-energy $x_{\text {soe }}(t)$, and the polarization voltage, $V_{F, \text { soe }}(t)$ as a state vector

$$
x(t)=\left(\begin{array}{c}
x_{\text {soe }}(t) \\
V_{F, \text { soe }}(t)
\end{array}\right)
$$

As we found in literature, there are different concepts on energy and definition for SoE. Here we have listed a couple of definition for SoE.

As [13] the available energy of the battery is integration over time of the effective power as

$x_{\text {soe }}(t)=x_{\text {soe }}\left(t_{0}\right)+\int_{0}^{\tau} P_{\text {eff }} d \tau$

Where $P_{e f f}$ is effective power of the battery. However, the authors did not specified the definition of $P_{\text {eff }}$, where it is function of terminal voltage or internal opencircuit voltage of the battery. The $P_{\text {eff }}$ relates to the requested power $P_{B}$ of the $\mathrm{EV}$ and power loss $P_{\text {loss }}$ as

$P_{B}=P_{\text {loss }}+P_{\text {eff }}$

Alternatively, the literure [18] defined battery energy similar to (15) except relation of SoC and energy. In [18] the battery power expressed as sum of power stored $P_{S}$ in the battery and power loss $P_{\text {loss }}$ as

$P_{B}=P_{\text {loss }}+P_{s}$
Since $P_{\text {loss }}$ depends on the storage power $P_{s}$, the energy, and temperature $T$, the power loss modelled as quadratic of $P_{S}$. Thus battery power can be written as

$P_{B} \approx \beta P_{S}^{2}+P_{S}$

This work suggests that when power loss is negligible the battery power would be equal to effective power that same as storage power

$P_{B}=P_{\text {eff }}=P_{S}$

The stored power could be function of $V_{o c v}$, and its integration would lead to another definition of SoE. For example, in [15] the remaining discharge energy (RDE) was used to evaluate the energy storage of the battery

$$
\begin{aligned}
x_{\text {soe }}(t) & =\int_{t}^{t_{2}} V_{o c v} \cdot I_{L} I . d \tau=\int_{C_{t_{2}}}^{C_{t}} V_{o c v} d I_{L} \tau \\
& =C_{B} \int_{S o C_{t_{2}}}^{S o C_{t}} V_{o c v} d S o C
\end{aligned}
$$

The assuming the energy of the battery as a useful work at terminal of the battey the SoE is defined as [14]

$x_{\text {soe }}(t)=x_{\text {soe }}\left(t_{0}\right)+\int_{0}^{\tau} \frac{V_{T}(t) I_{L}}{C_{B}} d \tau$

Where $x_{\text {soe }}(t)$ is the state-of-charge at time $\mathrm{t}, x_{\text {soe }}\left(t_{0}\right)$ is the SoE at the time $t_{0}, I_{B}$ is the battery current and $C_{B}$ is the capacity of battery.

The required battery power for an aerospace vehicle can be wrttien as

$P_{B}=\frac{v D}{\eta_{\text {motor }} \eta_{\text {prop }}}+P_{\text {payload }}+P_{\text {avionics }}$

Where $v$ and $D$ is the freestream velocity and the total air drag, respectively, of the air vehicle, $\eta_{\text {motor }}$ is the powertrain motor efficiency, $\eta_{\text {prop }}$ is the propeller efficiency, $P_{\text {payload }}$ is the power for payload and $P_{\text {avionics }}$ is the power for avionics.

In this work we assume that stored power $P_{S}=V_{o c v} I_{L}$ as effective power no-loss in power and maximum (unit) efficiency, the $P_{S}$

$x_{\text {soe }}(t)=x_{\text {soe }}\left(t_{0}\right)+\frac{1}{E_{B}} \int_{0}^{\tau} V_{o c v}(S o E) I_{L} d \tau$

Where $E_{B}$ is the energy capacity of the battery cell, this is not same as the total capacity of battery cell $C_{B}$.

Since this paper uses discrete EKF, the discrete time, the discreteequation (9) can be expressed in discrete-time as

$x_{\text {soe }}(k)=x_{\text {soe }}(k-1)+\frac{V_{o c v, s o e}(k) I_{L}(k) \Delta t}{E_{B}}$

For estimate SoE using the EKF, the measurement function can be written as

$y_{\text {soe }}(k)=h\left(x_{\text {soe }}(k), V_{F, \text { soe }}(k), I_{L}(k)\right)+v(k)$

where

$h()=.V_{T, \text { soe }}=V_{\text {ocv }}(\mathrm{soe})-V_{F}(\mathrm{soe})-V_{i}(k)$

is terminal voltage of battery that modelled from ECN as shown in Fig 5. For SoE estimation problem, the Li-S battery cell is modelled as Thevenin's equivalent circuit network model as shown in Fig.

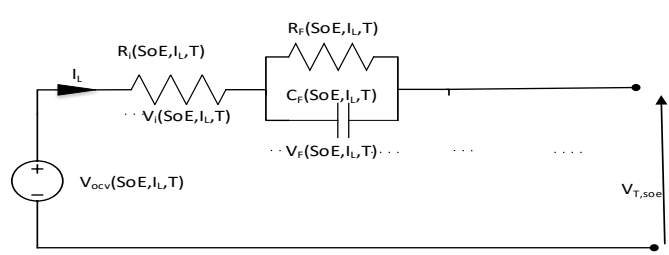

Fig.5 Battery model for SoE estimation 


\section{Battery Parameter Estimation}

The battery parameters are estimated using prediction error minimisation(PEM) approach as $[1,3]$. The parameters of cell represented can be expressed as new behaviour variables as [3]. We have battery system state vector $x(t)$. Assume that predicted state is $\hat{x}(t)$ and have initial condition of state $\hat{x}(t)$. Dynamic the state SoC and SoE

These new parameters have to estimated using PEM. The parameter vector is defined as

$\theta=\left[V_{\text {ocv }}, R_{\text {int }}, \Omega, \rho\right]^{\prime}$

here $^{\prime}$ is the transpose operator.

Let $y_{c}(k)$ and $\hat{y}_{c}(k)$ is the measured and estimated voltage for $k=0,1, \ldots, t$. From equation (), the estimated

$\hat{y}(k)=h((\hat{x}(k) \mid \theta))=h\left(\begin{array}{c}\hat{x}_{\text {soc }}(k) \\ \left(\hat{V}_{F, \text { Soc }}(k) \mid \theta\right)\end{array}\right)$

Defining prediction error

$\varepsilon=y(k)-\hat{y}(k)$

The PEM minimizes the cost function $J(\theta)$ as

$\varepsilon \max _{\theta, \hat{x}(0)} J(\theta)=\operatorname{trace}\left(\sum_{k=1}^{t} \varepsilon \varepsilon^{\prime}\right)$

Subject to

$x(k+1)=f(x(k), u(k)) ; k=0,1, \ldots t-1$

with $x(0)$ is the initial condition. The boundary condition on parameters is given as $\theta_{i, \min }<\theta_{i}<$ $\theta_{i, \max }$, in which $i=1,2,3,4$ denote parameter vector index, and $\theta_{i, \min }$ and $\theta_{i, \max }$ represent respectively, maximum and minimum limit of value of parameter.

\section{The EKF based SoC and SoE Estimation}

The EKF is a conventional, however widely accepted by control community, recuressive nonlinear estimation algorithm. The time and measurement update recursively as shown in Fig.

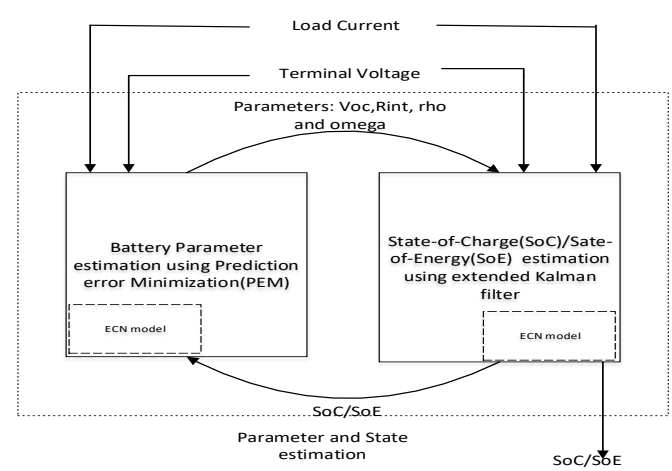

Fig.6 The recursive process of EKF while estimating SoC and $\mathrm{SoE}$

The EKF uses first-order approximation of taylor series, is also known as Jacobian of nonlinear functions. In this problem, the Jacobain for state equation is evaluated as

$F=\left[\begin{array}{ll}\frac{\partial f_{\text {soe }}}{\partial x_{\text {soe }}} & \frac{\partial f_{\text {soe }}}{\partial V_{F}} \\ \frac{\partial f_{V}}{\partial x_{\text {soe }}} & \frac{\partial f_{V}}{\partial V_{F}}\end{array}\right]$
And measured voltage is linearised with following Jacobain

$H=\left[\begin{array}{ll}\frac{\partial h(.)}{\partial x_{\text {soe }}} & \frac{\partial h(.)}{\partial V_{F}}\end{array}\right]$

Thus the nonlinear state and measurement models, which are functions of the $\mathrm{SoC} / \mathrm{SoE}$ are linearized. While estimating the SoC and SoE, the priori state estimate and Jacobians of EKF evaluate as shown in fig. 6 . The EKF switches the polynomial fitting model of battery parameters based a value determined from voltage vs SoC, as shown in fig 7. curve while estimating SoC and SoE of low and high-plataeu.

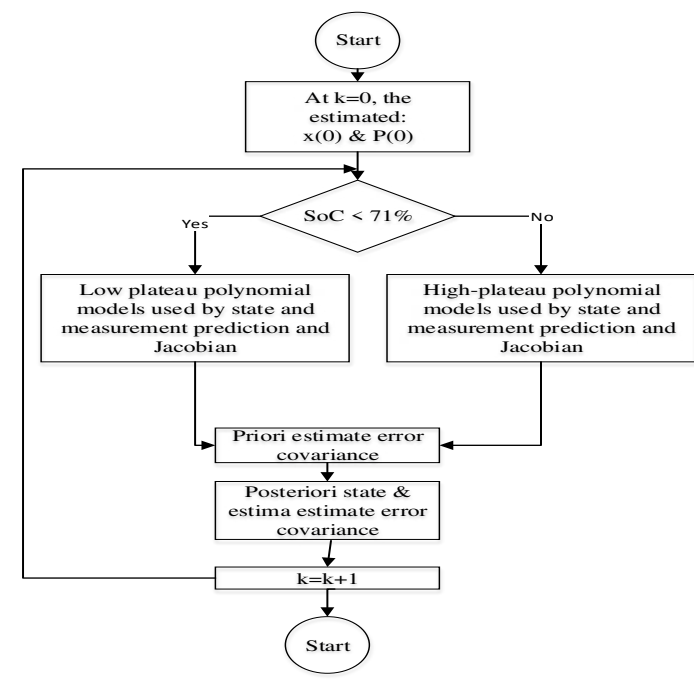

Fig.6 Flowchart for low- and high-plateau polynomial models in EKF.

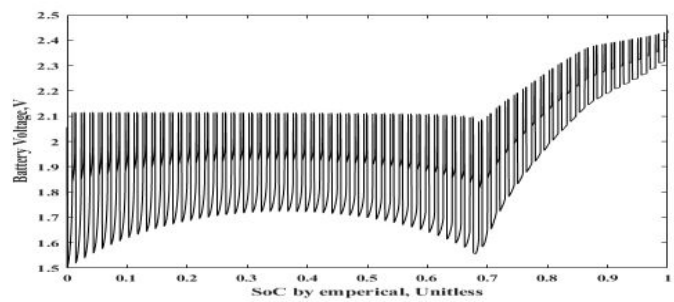

Fig.7 The empirical SoC vs battery voltage curve.

Since the EKF uses analytical approximation of nonlinear functions, the investigating the performance of estimation could help to tune the parameter so that the estimation accuracy would be improved. For investigate the performance of EKF, an analysis of the degree of observability approach can be used [17] .

In this work, the following observability matrix is used for DoO analysis of both SoC and SoE estimation by EKF:

$M(k)=\left[\begin{array}{c}H(k) \\ H(k) F(k)\end{array}\right]$

Defining the condition as a ratio between maximum and minimum of singular number of observability matrix $M(k)$

$\operatorname{cond}(M(k))=\frac{\max (M(k))}{\min (M(k))}$

the degree of observability

$\operatorname{DoO}(k)=\frac{1}{\operatorname{cond}(M(k))}$ 
The DoO the measures of local observability, which is used to investigate the dependability of an estimation.

\section{Results}

The SoE estimation by the EKF, and comparison of SoE to SoC are demonstrated with MATLAB simulation results. In this simulation, the SoE and SoC for the discharge test data of the sample cell, which is given Fig 1, is presented. While estimating SoC and SoE, the cofficients of polynomial fitting models for parameters given in Table 1 are used for priori state and measurement, and corresponding Jacobians at low and high-plateau appropriately. For instance, the open circuit voltage $8^{\text {th }}$ order polynomial model

$$
\begin{gathered}
V_{\text {oc }}(\text { soe })=-752.62 x_{\text {soe }}^{8}+2085 x_{\text {soe }}^{7}-2392.87 x_{\text {soe }}^{6} \\
+1466.98 x_{\text {soe }}^{5}-517.42 x_{\text {soe }}^{4}-105.21 x_{\text {soe }}^{3} \\
\quad-11.69 x_{\text {soe }}^{2}+0.62 x_{\text {soe }}^{1}+2.1
\end{gathered}
$$

is used for low-plateau SoE estimation and whereas the $5^{\text {th }}$ order polynomial is

$$
\begin{gathered}
V_{\text {oc }}(\text { soe })=108.1 x_{\text {soe }}^{5}-361.13 x_{\text {soe }}^{4}+444.73 x_{\text {soe }}^{3} \\
-238.18 x_{\text {soe }}^{2}+47.03 x_{\text {soe }}+1.88
\end{gathered}
$$

\begin{tabular}{|c|c|c|c|c|}
\hline plateau & Voc & Rint & Rho & omega \\
\hline low & $\begin{array}{l}{[-752.62} \\
2085.66 \\
-2392.87 \\
1466.98 \\
-517.42 \\
105.21 \\
-11.69 \\
0.62 \\
2.1]\end{array}$ & $\begin{array}{l}{[3.792} \\
-8.212 \\
6.428 \\
-1.56 \\
0.07346 \\
0.1458]\end{array}$ & $\begin{array}{l}{[0.5087} \\
-0.6572 \\
0.6815]\end{array}$ & $\begin{array}{l}{[6.8270} \\
-7.4220 \\
1.6850 \\
0.1407 \\
-0.1544 \\
0.1441]\end{array}$ \\
\hline high & $\begin{array}{l}{[108.1} \\
-361.13 \\
444.73 \\
-238.18 \\
47.03 \\
1.88]\end{array}$ & $\begin{array}{l}{[1.07} \\
-2.445 \\
1.457]\end{array}$ & $\begin{array}{l}{[-0.0451} \\
0.4892]\end{array}$ & $\begin{array}{l}{[0.415} \\
-0.7441 \\
0.3469]\end{array}$ \\
\hline
\end{tabular}

used for high-plateau SoE estimation. Similarly the $\boldsymbol{R}_{\text {int }}, \boldsymbol{\Omega}$, and $\boldsymbol{\rho}$ have different order for low and high-plateau sepereately and their coefficients are listed in the Table 1.

Table 1 The coefficients of parameter polynomial models

The estimated SoE and polarisation voltage obtained by SoE EKF is shown in Fig 8a and 8b, respectively. In Fig 8a, the yaxis scale 1 denotes $100 \%$ and 0 denotes $0 \%$ of SoE. Since the test data is discharge, the initial SoE is very close to 1 and at end of discharge the $\mathrm{SoE}$ is close to 0 as expected. These result seem to similar trend of the SoC EKF. The perfromance of SoC EKF is shown Fig 9. The Fig 9a shows the estimated SoC and Fig.9b polarization voltage of cell. For given parameters and conditions the accuracy of SoE and SoC estimation by EKF in terms of the degree of observability is shown in Fig 10.
The range of the degree of observability of the $\mathrm{SoE}$ and $\mathrm{SoC}$ is $0<D o O<1$, which means the system for SoC and SOE estimation in BMS is locally observable entire period and estimators perform reasonable good. The DoO of SoE is close very close to $\mathrm{DoO}$ of $\mathrm{SoC}$ and this indicates that $\mathrm{SoE}$ of $\mathrm{Li}-\mathrm{S}$ is very close SoC. It is worthy to analyse that at $70 \% \mathrm{SoE}$ and SoC, the EKF switches from the high- to low-plateau, and then follow ups slowly to reache the $10 \%$. The perfromance over $10 \%$ to $0 \%$ is almost flat, but not bad. However, this work need further investigation to improve the accuracy in estimation at low-plateau region. By tuning parameters the performance of low-plateau could be improved. In both $\mathrm{SoE}$ and $\mathrm{SoC}$ estimation, at $70 \% \mathrm{SoE} / \mathrm{SoE}$ the the performance of EKF at high-plateau is more accurate than the low-plateau.

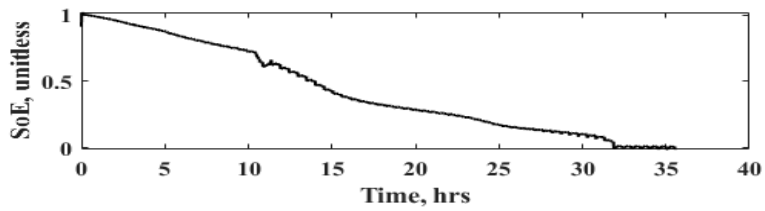

(a)

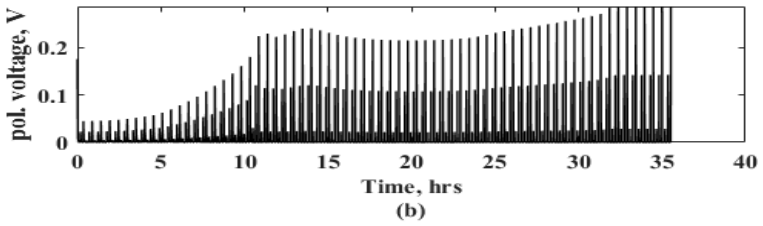

Fig.8 The performance of SoE EKF (a) the estimated SoE (b) estimated polarization voltage
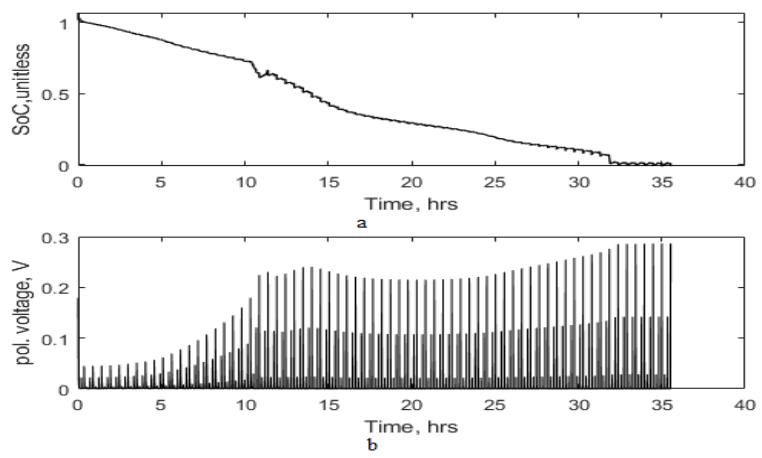

Fig.9 The performance of SoC EKF (a) the estimated SoC (b) estimated polarization voltage

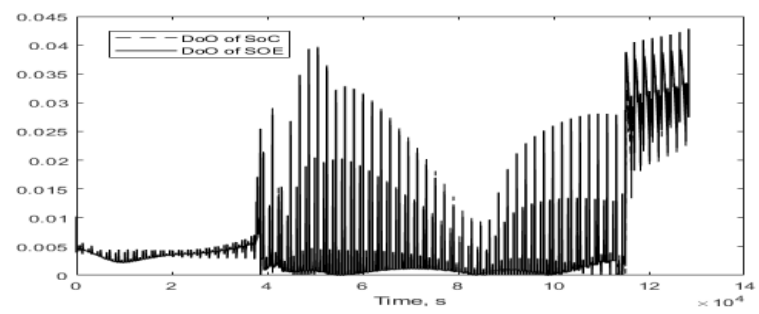

Fig.10 the degree of observability of EKF while estimating $\mathrm{SoC}$ and $\mathrm{SoE}$ 

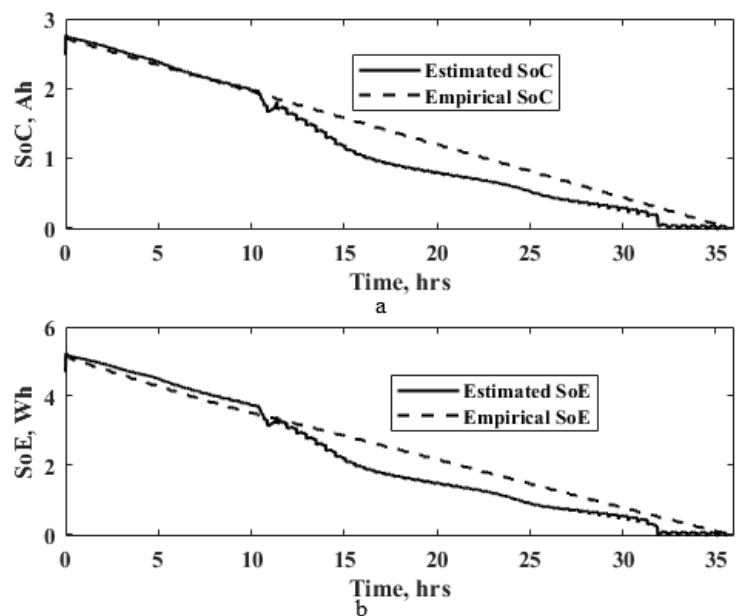

Fig.11 With unit, estimated and empirical comparison: (a) SoC and (b) SoE

Figure 11 shows the estimated SoC in terms of Ah (Ampherehour) and SoE in terms of Wh (Watt-hour), and its comparison with empirical result. The squared error (Fig 12) between estimated and empherical one which can be observed in DoO analysis Fig 10 as well. Fig12a shows the error for SoC, and Fig12b for SoE. In both cases, the accuracy of high platea is better than the low plateau. In overall, the squared error for SoC estimation by the EKF is more than 0.03 (3\%), whereas the SoE estimation error by is $2.5 \%$, whic less than that of SoC. The estimation accuracy of EKF in the high plateau region is more than the low plateau. Moreover, the error for both SoC and SoE estimation is less than 5\%, which is quite sensible and acceptable.

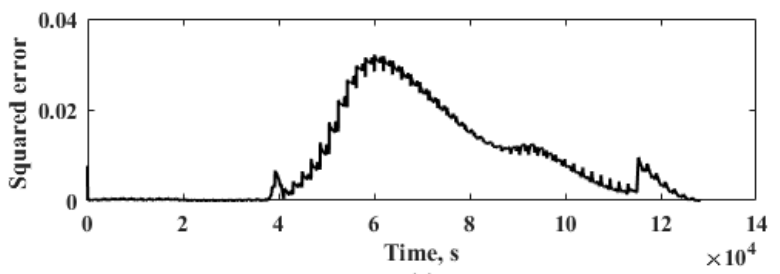

(a)

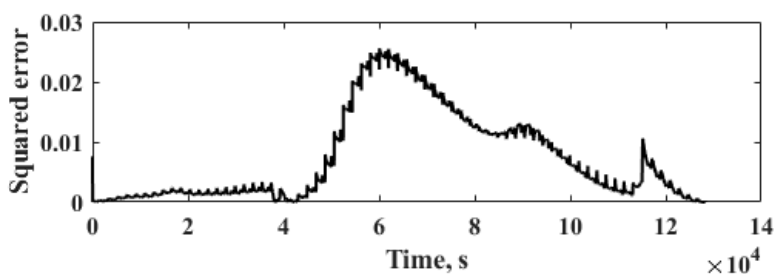

(b)

Fig.12 The squared error : (a) SoC and (b) SoE

\section{Conclusion}

The SoE estimation of Li-S battery is formulated and it has been estimated using extended Kalman filter. In order to estimate Li-S battery parameters, the prediction error minimization is used. Assuming particular conditions and polynomial curve fitting models of parameters for high- and low-plateau, the EKF estimates SoE, and compared that to the SoC. Using degree of observability analysis, the estimation accuracy of SoE and SoC have been investigated. Having test result of discharge profile obtained by experiment, the SoE estimation is demonstrated and that compared to the SoC. Resuls show that though mathematically the SoE and SoC problems are differtent, the SoE and SoC estimation of Li-S battery by the EKF is similar. In both SoC and SoE estimation by EKF, the estimation error is $5 \%$, which is acceptable.

\section{Acknowledgements}

The authors thank to the Aerospace Technology Institute (ATI) and Innovate UK who funded this work under grants TS/P003818/1 and TS/R013780/1.

Research data is currently embargoed for commercial reasons. For further details, please contact researchdata@cranfield.ac.uk.

\section{References}

[1] Karsten, P., Daniel J. A., Abbas, F, Stefano, L., Vadav, K. : 'Kalman-variant estimators for state of charge in Lithiumsulfur batteries', Journal of Power Sources, 2017, 343, pp. 254-267

[2] Karsten, P., Daniel J. A., Abbas, F, Monica, M., Vaclav, K, Stefano, L. : 'Improved State of Charge Estimation for Lithium-Sulfur Batteries', Journal of Energy Storage, 2019, 26, pp.

[3] Propp K, Marinescu M, Auger DJ, O'Neill L, Fotouhi A, Somasundaram K, Offer GJ, Minton G, Longo S, Wild M \& Knap V (2016) Multi-temperature state-ependent equivalent circuit discharge model for lithium-sulfur batteries, Journal of Power Sources, 328 (October) 289-299. Dataset/s: 10.17862/cranfield.rd.c.3292031

[4] Fotouhi A, Auger DJ, Propp K, Longo S, Purkayastha R, O'Neill L \& Walus S (2017) Lithium-Sulfur cell equivalent circuit network model parameterization and sensitivity analysis, IEEE Transactions on Vehicular Technology, 66 (9) 7711-7721.

[5] Knap V, Auger DJ, Propp K, Fotouhi A \& Stroe D-I (2018) Concurrent real-time estimation of state of health and maximum available power in lithium-sulfur batteries, Energies, 11 (2133) 1-23.

[6] Fotouhi A, Auger D, Propp K \& Longo S (2018) Lithiumsulfur battery state-of-charge observability analysis and estimation, IEEE Transactions on Power Electronics, 33 (7) 5847-5859.

[7] Antaloae C, Marco J \& Assadian F (2012) A novel method for the parameterization of a $\mathrm{Li}$-ion cell model for $\mathrm{EV} / \mathrm{HEV}$ control applications. IEEE Transactions on Vehicular Technology, 61(9), 3881-3892. https://doi.org/10.1109/TVT.2012.2212474

[8] Daniel J. Auger, Srinivasam Munisamy and Abbas Fotouhi, How accurate is state of charge as a predictor of remaining useful work?, 2019 International conference on Lithium-Sulfur Batteries, Aug 12-15, 2019, Beijing,China. 
[9] K. Li and K. J. Tseng, An equivalent circuit model for state of energy estimation of lithium-ion battery 2016 IEEE Applied Power Electronicns Conference and Exposition (APEC), March 2016Marchl, pp3422-3430

[10] Jiale Xie and Jiachen Ma and Jun Chen, Avaliable power prediction limited by multiple contraints for $\mathrm{LiFePO} 4$ batteries based on central difference Kalman filter, International Journal of Energy Research, 42, pp 4730-4745, 2018.

[11] Hicham Chaoui, Navid Golbon, Imad Hmouz, Ridha Souissi, Sofience Tahar, Lyapunov-based adaptive state of charge and state of health estimation for Lithium-ion batteries, IEEE transactions on industrial electronics, Vol 62, No.3, March 2018, pp: 1610-1618.

[12] Lennart Ljung, System Identification: Theory for the User, Second Edition, Prentice Hall, Dec 1998.

[13] Clemens Guenther, Benjamin Schott, Wildried Hennings, Paul Waldowski, Micheal A Danzer, Model-based investigation of electric vehicle battery ageing by mean of vehicle-to-grid scenario simulation, Vol. 239, 2013, pp: 604610

[14] HongWen He, YongZhi Zhang, Rui Xiong, Chun Wang, A novel Gaussian model based battery state estimation approach: State-of-Energy, Vol. 151, 2015, pp

[15] Xu Zhang, Yujie Wang, Chang Liu, Zonghai Chen: A novel approach of remaining discharge energy prediction for large format Lithium-Ion battery pack, Journal of Power cource, 343, 2017, pp:216-225.

[16] Stefen Leutenegger, Unmanned Solar Airplanes: design and algorithms for efficient and robust autonomous operation, Doctor of Sciences of ETH Zurich, 2014.

[17] Jannatun Nahar, Jinfeng Liu, SirishL. Shah: Parameter and state estimation of an agro-hydrological system based on system observability analysis, Computers and Chemical Engineering 121 (2019) 450-464.

[18] M. Koot and J. T. B. A. Kessels and B. de Jager and W. P. M. H. Heemels and P. P. J. van den Bosch and M. Steinbuch, Energy management strategies for vehicular electric power systems, IEEE Transactions on Vehicular Technology, march 2005, 54(3),pp 771-782. 
Paper Amendment

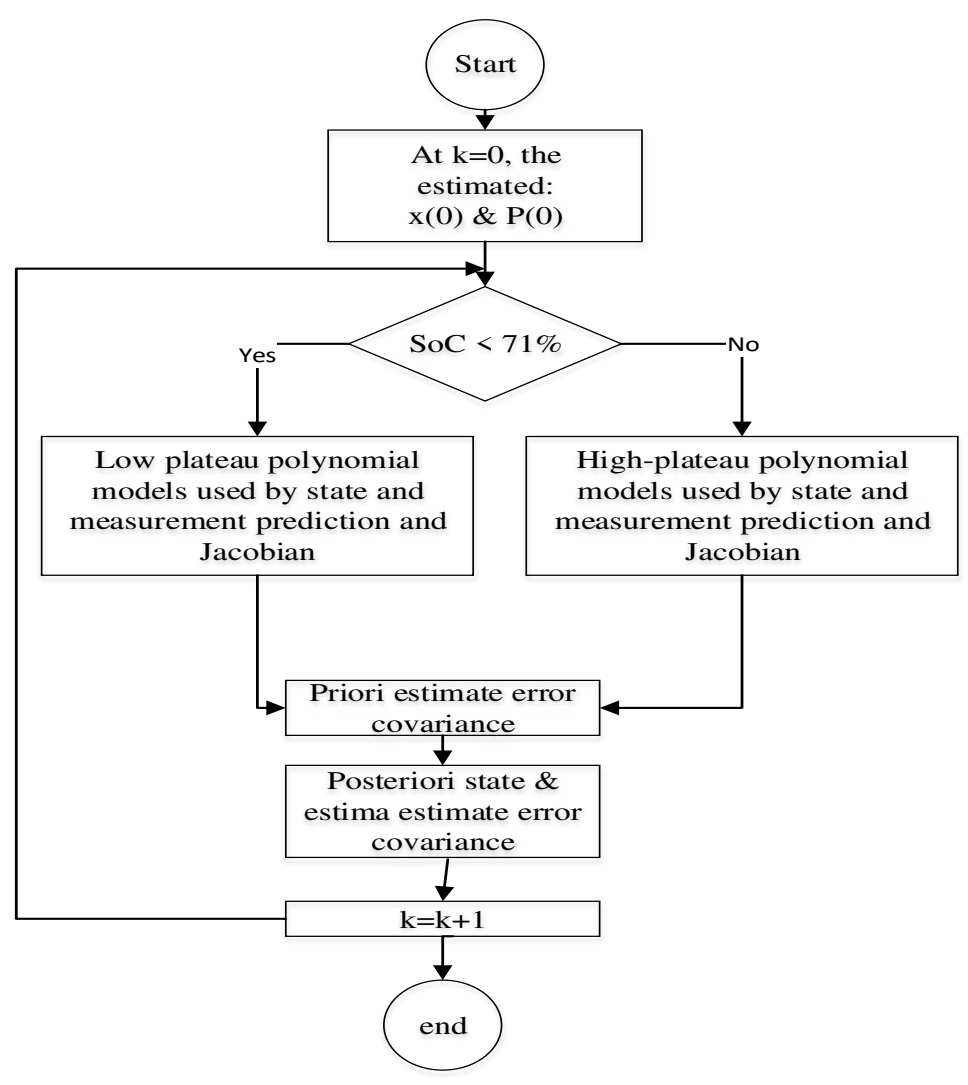

Fig.6 Flowchart for low- and high-plateau polynomial models in EKF. 
2020-12-03

State of energy estimation in electric propulsion systems with lithium-sulfur batteries

Munisamy, Srinivasan

Institution of Engineering and Technology (IET)

Munisamy S, Auger DJ, Fotouhi A. (2020) State of energy estimation in electric propulsion systems with lithium-sulfur batteries. In: 10th IET International Conference on Power Electronics, Machines and Drives (PEMD2020), 15-17 December 2020, Virtual Event https://doi.org/10.1049/icp.2021.1187

Downloaded from Cranfield Library Services E-Repository 